Particle Accelerators, 1990, Vol. 28, pp. 213-218 Reprints available directly from the publisher Photocopying permitted by license only
(C) 1990 Gordon and Breach, Science Publishers, Inc. Printed in the United States of America

\title{
THE HIGH FIELD SUPERFERRIC MAGNET II
}

F. RUSSELL HUSON, JOHN C. COLVIN, WILLIAM W. MACKAY, PETER M. MCINTYRE, SERGIO PISSANETZKY, RICHARD ROCHA, WILLIAM M. SCHMIDT, GARRY SHOTZMAN, AND JOHN C. ZEIGLER Texas Accelerator Center 4802 Research Forest Drive, Bldg. 2, The Woodlands, Texas 77380

Abstract A high-field superferric accelerator dipole has been built and tested. The magnet was designed to operate at $7.6 \mathrm{Tesla}$. It achieved $6.5 \mathrm{Tesla}$ with no training. The reduced performance is due to inadequate preload of the magnet during cooldown, and can be remedied by modification of the outer lining of the magnet.

\section{INTRODUCTION}

The Texas Accelerator Center (TAC) has conducted a systematic development of superferric accelerator dipole magnets over the past five years. The superferric design ${ }^{1}$ is characterized by a close coupling of the superconducting coil to the iron flux return of the magnet. The coil is arranged in a rectangular package rather than the $\cos \theta$ geometry which is required when the iron flux return is decoupled from the coil region. The benefits of the superferric design are

- the rectangular coil package and flux return are easy to manufacture and maintain dimensional control;

- the Lorentz forces on the coil are reduced in magnitude and push the conductor directly against the side wall of the steel flux return, providing for straightforward preloading of the coil to prevent coil motion and training;

- differential contraction of a stainless-steel outer lining can be used to deliver preload only while the magnet is cold, eliminating creep of coil insulation while at room temperature; 
- the iron flux return significantly reduces the magnetic reluctance, and hence reduces the amount of superconductor required to achieve a given field.

A year ago TAC successfully tested its first two-mode superferric magnet. ${ }^{2}$ In the two-mode magnet, the rectangular coil is segmented into two current circuits which are separately energized. The outer circuit is a simple rectangular coil which functions as a classic Panofsky window-frame magnet at fields up to iron saturation ( $\sim 2 \mathrm{Tesla}$ ). The inner circuit, also composed of rectangular coil segments, is arranged to approximate the $\cos \theta$ distribution required as $\mu \rightarrow 1$ at high field. By current programming the two circuits an excellent dipole field quality can be achieved at all excitations over a 20:1 dynamic range. ${ }^{3}$

The first two-mode superferric magnet was designed for 6 Tesla operation ( $90 \%$ of short-sample limit). It achieved 5.85 Tesla, exhibited no training, and had excellent field homogeneity. During the past year TAC has designed and built a second two-mode superferric magnet, designed for 7.6 Tesla operation. This paper presents the design, the test results, and an analysis of their significance.

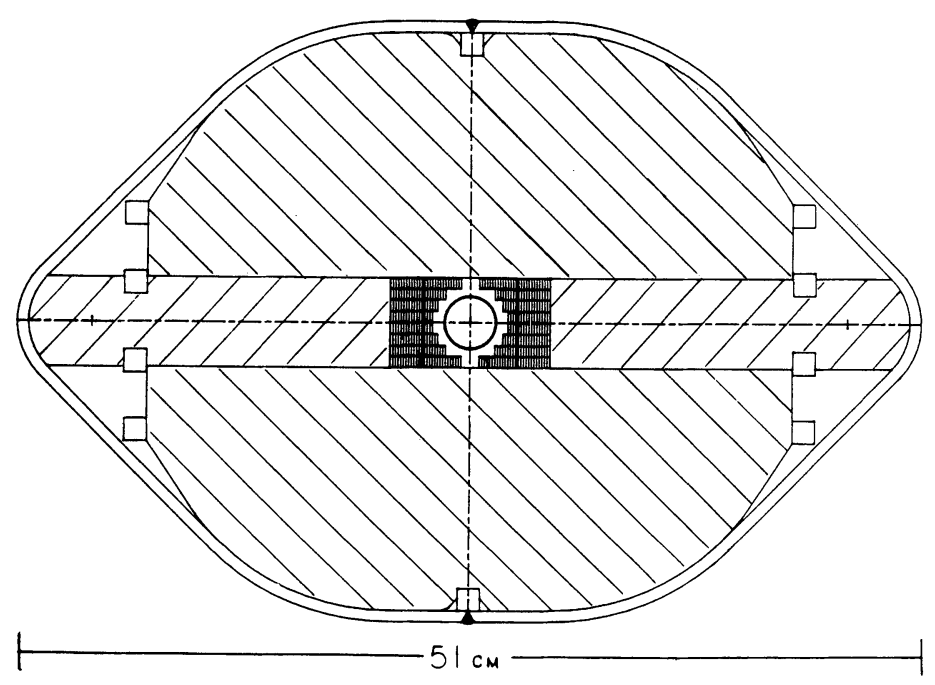

FIGURE 1 Cross-section of two-mode superferric magnet. 
DESIGN

The magnet is shown in cross-section in Figure 1. It consists of a two-circuit rectangular coil package, a beam tube/coil mandrel assembly, and a 4-component flux return. Figure 2 shows the current programing of the two circuits which is calculated to produce homogeneous dipole field over the operating range of field strength.

The magnet flux return is designed to accommodate the delivery of a horizontal preload to the coil which can counter the Lorentz force on the coil in operation and thereby prevent coil motion. This is achieved by segmenting the flux return into 4 rectangular blocks as shown in Figure 1, so that the two side "plungers" can slide under preload within the two large blocks. The blocks and plungers are each fabricated as welded stacks of laminations in the usual fashion. These stacks must then slide upon one another in the plane of the laminations.

We experimentally verified that this indeed occurs and measured the coefficient of friction under relevant transverse loading at cryogenic temperatures. Figure 3 shows the coefficient of static friction as a function of loading force.

A horizontal preload $\vec{F}_{p}$ is applied to the coil through differential contraction of the outer lining. The stainless steel lining shrinks more during cool-down than the iron flux return, resulting in a tension $\mathrm{T}$ :

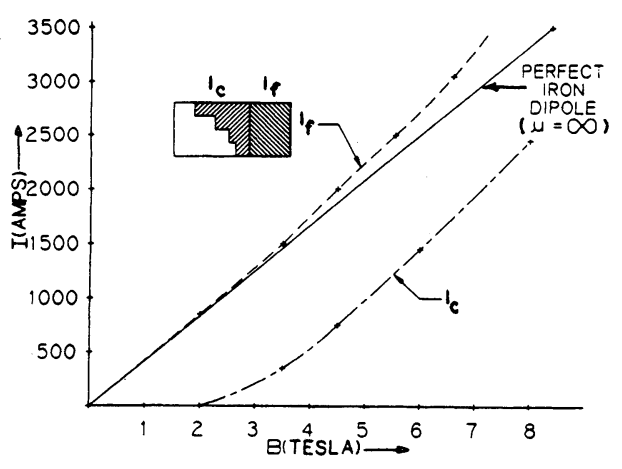

FIGURE 2 Calculated current programming of the two coil segments.

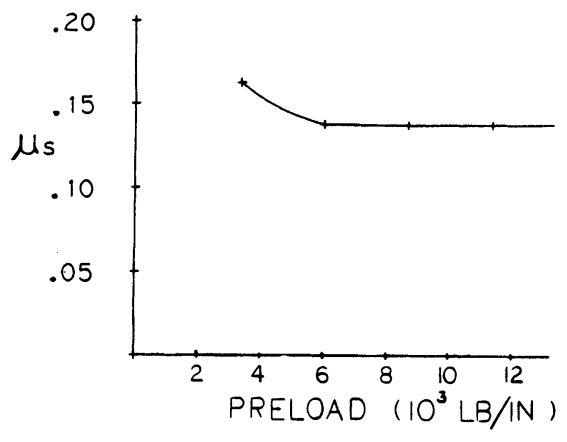

FIGURE 3 Measured coefficient of static friction for lamination stacks. 
$\mathrm{T}=\mathrm{Y} \mathrm{t} \Delta \ell / \ell$

where $Y=30 \mathrm{Mpsi}$ is the Young's modulus

$t=0.63 \mathrm{~cm}$ is the lining thickness

$\Delta \ell / \ell=10^{-3}$ is the cumulative differential contraction.

The stress in the outer lining produces equal horizontal and vertical preloads within each quadrant $F_{p}=T / \sqrt{2}$ on the plungers and blocks respectively.

The various forces acting on the coil assembly and flux return are shown schematically in Figure 4. The preload was designed to exceed the horizontal Lorentz force $F_{c x}$ at maximum design field and thereby prevent coil motion at all operating field strengths. This consideration neglects several other forces, however, as will be discussed later.

The coil assembly was fabricated in the same fashion as in all previous superferric magnets. The conductor is 18 strand Rutherford cable $\left(.15 \times .62 \mathrm{~cm}^{2}\right)$, with 2 half-wraps of $25 \mu \mathrm{m}$ Kapton insulation. The superconductor strand is composed of 2000 NbTi filaments, $9 \mu \mathrm{m}$ diameter, imbedded in a copper matrix to a ratio $1.8: 1$. The measured short-sample current is $2896 \mathrm{~A}$ at 5 Tesla.

\section{MAGNET TESTS}

The magnet attained a field of 6.50 Tesla. On successive quenches, the peak field was $6.48,6.49,6.50,6.50$ Tesla: there was no training. From short-sample measurements we had predicted a peak field of 7.6 Tesla.

The measured and calculated multipoles are given in Table I. For the two lowest excitations, the design configuration called for $I_{c}=0$. We were unable to deliver less than $10 \mathrm{~A}$ using the power supplies in the test configuration. This produced the large higher multipoles observed in the three lowest excitations. The significant and relatively constant value of $a_{1}$ indicates an off-center displacement of the coil package within the flux return assembly. 
LORENTZ FORCES, FRICTION, AND COIL MOTION

To understand the disappointing reduction in magnet quench performance below the short sample limit, we must return to the analysis of the forces shown in Figure 4. For one quadrant,

$-F_{p x}=F_{p y}=F_{p}=5300 \mathrm{lb} /$ in horizontal, vertical preload

$\mathrm{F}_{\mathrm{Sx}}=-165 \mathrm{lb} / \mathrm{in}$ horizontal Lorentz force on steel plunger

$\mathrm{F}_{\text {sy }}=2600 \mathrm{lb} / \mathrm{in}$ vertical Lorentz force on steel block

$\mathrm{F}_{\mathrm{cx}}=3700 \mathrm{lb} / \mathrm{in}$ horizontal Lorentz force on coil assembly

$\mathrm{F}_{\text {cy }}=1630 \mathrm{lb} / \mathrm{in}$ vertical Lorentz force on coil assembly The values given for $\vec{F}_{S}$ and $\vec{F}_{c}$ correspond to the observed quench field of 6.5 Tesla. There are two other forces acting on the assembly: static friction $F_{f}$ at the interface between the block and plunger, and compression $\mathrm{F}_{\mathrm{m}}$ of the coil mandrel.

The initial preload is delivered with no magnetic excitation $\left(\vec{F}_{S}=\vec{F}_{c}=0\right)$.

Equilibrium requires that

$$
\begin{aligned}
& F_{p x}+F_{f}+F_{m}=0 ; \\
& F_{f}=+\mu F_{p} \\
& F_{m}=(1-\mu) F_{p}
\end{aligned}
$$

At full excitation, the net force on the steel plunger is reversed and is acting to move the coil outwards. Coil motion will occur if the net force exceeds the static friction:

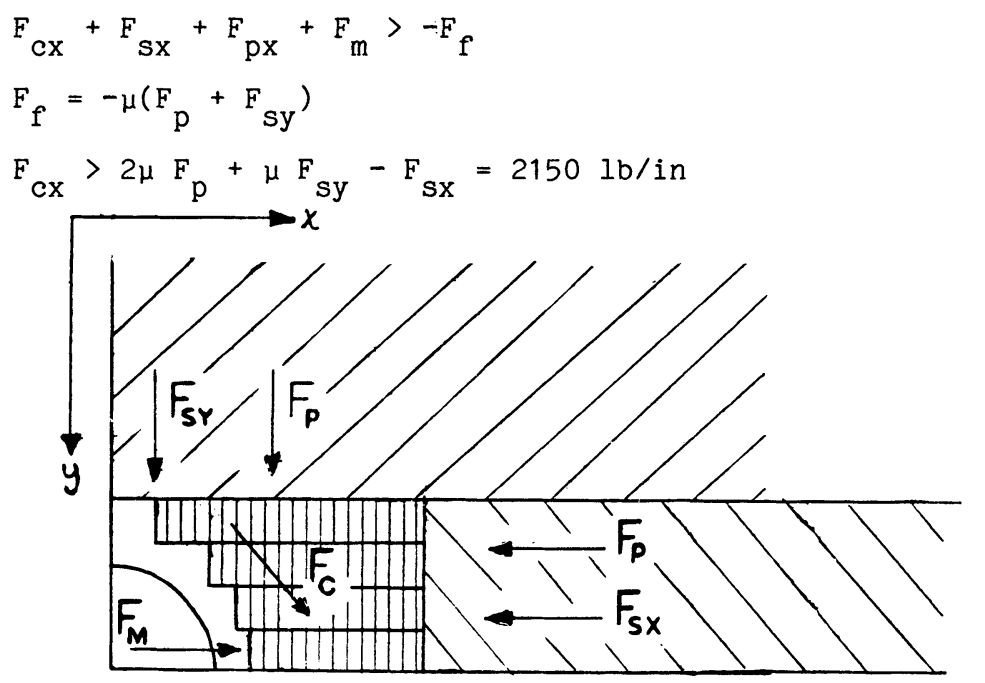

FIGURE 4 Diagram of component forces acting on the coil assembly and flux return. 
Coil motion will therefore result even below the 6.5 Tesla where quench is observed, and quench finally occurs when the coil motion results in a superconductor element exceeding critical current in the presence of trapped flux. Our choice of preload was governed by the erroneous consideration that $F_{p}>F_{c x}$ would prevent coil motion.

This undesirable state of affairs can be remedied by doubling the thickness of the outer lining, hence doubling $F_{p}$. No sliding will then occur up to a field of 7.7 Tesla, beyond short sample limit. The test magnet will be reassembled with a suitable outer lining and retested. It is interesting to note that, even when a superferric magnet is erroneously assembled with inadequate preload, the quench field is well-defined and does not train.

TABLE I Measured (and calculated) harmonics ( $\mathrm{cm}^{-n}$ )

\begin{tabular}{llllllll}
\hline$I_{F}(A)$ & $I_{C}(A)$ & $B_{0}(T)$ & $b_{1}$ & $a_{1}$ & $b_{2}$ & $b_{3}$ & $b_{4}$ \\
\hline 100 & $10(0)$ & 0.25 & -0.4 & 2.0 & $-78(-0.4)$ & -0.6 & $3.7(0.4)$ \\
200 & $10(0)$ & 0.49 & -2.0 & -1.5 & -37 & -0.5 & 2.2 \\
400 & $10(0)$ & $0.96(1.00)$ & -2.0 & -2.1 & $-19(-0.4)$ & 0.4 & $1.9(-0.1)$ \\
833 & 12 & $1.98(2.01)$ & -3.0 & -3.4 & $-9.5(-0.9)$ & -0.5 & $1.8(0.1)$ \\
1500 & 350 & $3.44(3.45)$ & -3.8 & -4.3 & -2.2 & -0.6 & $1.7(0.5)$ \\
2000 & 750 & $4.46(4.50)$ & -2.9 & -5.2 & $0.4(-0.3)$ & -1.0 & $1.9(0.1)$ \\
2500 & 1200 & $5.48(5.51)$ & -2.6 & -4.7 & $0.5(0.7)$ & -1.3 & $1.2(-0.3)$ \\
2800 & 1450 & $6.05(6.13)$ & -3.3 & -4.7 & $2.5(-0.8)$ & -0.8 & $0.2(-0.5)$ \\
\hline
\end{tabular}

\section{REFERENCES}

1. F.R. Huson, et al., "Superferric Magnet Option for the SSC," 1985 Particle Accelerator Conference, (invited paper), Vancouver, B.C., May 1985.

2. J.C. Colvin, et al., "The High-Field Superferric Magnet," Nucl. Inst. and Meth. in Phys. Res. A270, 207-211 (1988).

3. W. Xie, et al., "A High-Field Two-Mode Superferric Magnet," 1986 Applied Superconductivity Conference, Baltimore, Maryland, September 1986. 